\title{
Rapid liquid chromatography-tandem mass spectrometry method for the determination of a broad mixture of pharmaceuticals in surface water
}

\author{
Justin M. Conley ${ }^{\mathrm{a}, *}$, Steven J. Symes ${ }^{\mathrm{b}}$, Scott A. Kindelberger ${ }^{\mathrm{b}}$, Sean M. Richards ${ }^{\mathrm{a}}$ \\ ${ }^{a}$ Department of Biological and Environmental Sciences, University of Tennessee at Chattanooga, 615 McCallie Avenue, Chattanooga, TN 37403, USA \\ ${ }^{\mathrm{b}}$ Department of Chemistry, University of Tennessee at Chattanooga, 615 McCallie Avenue, Chattanooga, TN 37403, USA \\ Received 24 October 2007; received in revised form 16 January 2008; accepted 22 January 2008 \\ Available online 31 January 2008
}

\begin{abstract}
Herein, a new method for the detection of 13 different pharmaceuticals and one metabolite in surface water at low ng/L levels is described. The method utilizes ultra performance liquid chromatography-tandem mass spectrometry and a solid-phase extraction sample preparation. Mean method detection limits were low $(4.10 \mathrm{ng} / \mathrm{L})$ and overall solid-phase extraction recovery and reproducibility was adequate (mean recovery, $77.9 \%$; mean RSD, 7.3\%). The method allows for quick run times and minimal solvent use as compared with other previously reported high performance liquid chromatography-based methods. Application of this method for the detection of pharmaceuticals in Tennessee River surface water determined that caffeine, sulfamethoxazole, and carbamazepine were frequently detected (100\% of samples). Trimethoprim was moderately detected ( $30 \%$ of samples); acetaminophen, atorvastatin, and lovastatin were infrequently detected (10\% of samples); and ciprofloxacin, diltiazem, fluoxetine, levofloxacin, norfluoxetine, ranitidine, and sertraline were not detected. This study reports the first detection of lovastatin in surface water. (c) 2008 Elsevier B.V. All rights reserved.
\end{abstract}

Keywords: Ultra performance liquid chromatography; Solid-phase extraction; Tandem mass spectrometry; Environmental analysis; Surface water; Pharmaceutical; Drug; Matrix effect; Standard addition

\section{Introduction}

It is well established that the source, presence, and fate of pharmaceutically active compounds in the aquatic environment is of concern [1-5]. This area of research has progressively received more attention as the ubiquity of many pharmaceuticals in surface water becomes evident. Currently more than 80 pharmaceutical compounds have been detected in sewage effluent, surface water, and groundwater in at least 12 different nations; the majority of studies were in Europe and North America [4,6]. A national U.S. reconnaissance study by Kolpin et al. [7] analyzed for pharmaceutical residues in surface waters of 33 states and smaller scale studies have been conducted in Iowa [8], Louisiana [9], and Nevada [10]. In each study, detectable levels of a variety of pharmaceuticals were reported. As of 2006, Tennessee is third in the nation for retail prescription drugs filled at

\footnotetext{
* Corresponding author.

E-mail address: justinmconley@ hotmail.com (J.M. Conley).
}

pharmacies at 15.8 per capita and 15 th in the nation for total population [11]. Despite the high per capita usage rate and relatively high population, no currently published studies have examined Tennessee waters for pharmaceutical contamination.

The toxicological effects of pharmaceuticals in the environment to humans and free living aquatic and terrestrial organisms are not well understood. Of particular concern are the potential impacts on aquatic organisms that may experience subtle, chronic life-cycle and multi-generational exposure to low doses of pharmaceuticals [2,5]. In order to elucidate these environmental and ecological impacts, there is a need for analytical methods that allow quantitation of pharmaceuticals in aquatic matrices $[2,3,5]$. Ideally these methods should be simple, rapid, sensitive, selective, broad spectrum, and able to overcome the negative effects of matrix components.

The most widely used instrumentation for determining concentrations of trace pharmaceuticals in environmental samples is liquid chromatography-tandem mass spectrometry (LC-MS/ MS) coupled with a solid-phase extraction (SPE) sample preparation step. The popularity of this procedure is due to the 
selectivity and sensitivity of LC-MS/MS and the ability of diverse SPE sorbent chemistries to bind a wide variety of molecules [12-14]. Numerous studies have successfully utilized LC-MS/MS to detect pharmaceutical residues in sewage treatment plant (STP) influent and effluent, surface water, and groundwater after sample pre-concentration using SPE [15-25]. Pharmaceutical residues are consistently analyzed down to the low ng/L level with drawbacks being matrix effects and separation difficulties for highly polar compounds [12].

The objective of this study was to develop a LC-MS/MS method for the determination of a broad spectrum of commonly used pharmaceuticals in surface water. Further, it was in the authors' interest to make the method as accurate, precise, simple, and cost-effective as possible without compromising sensitivity. To this end, a SPE sample pre-concentration coupled with a LC-MS/MS method for separation and detection of 13 different pharmaceuticals and 1 primary metabolite has been developed. The utility and ability of this method was applied to samples of surface water collected from the Upper Tennessee River Basin located in eastern Tennessee.

Selection of pharmaceutical compounds for method development was based on compounds previously used in a study on the effects of pharmaceuticals in aquatic mesocosms (acetaminophen, atorvastatin, caffeine, carbamazepine, ciprofloxacin, fluoxetine, levofloxacin, sertraline, sulfamethoxazole, and trimethoprim) [26,27]. Pharmaceuticals were chosen for that study based on the estimated usage rate inferred from the number of prescriptions dispensed per year in the U.S. [28], and commonly consumed non-prescription drugs (acetaminophen and caffeine). Diltiazem, lovastatin, and ranitidine were chosen based on ionization potential (electrospray positive compounds) and number of prescriptions dispensed per year in the U.S. [28]. Norfluoxetine was chosen because it is a primary metabolite of fluoxetine and has the potential to be more prevalent than its parent molecule. Overall, the goal was to have a variety of pharmaceuticals representing prescription and non-prescription classes. Selections for the method resulted in eight different major pharmaceutical classes being represented (analgesic, antibiotic, antiepileptic, antidepressant, antihypertensive, lipid regulator, stimulant, antihistamine) and one primary metabolite (Table 1).

\section{Experimental}

\subsection{Pharmaceutical standards and reagents}

All pharmaceutical compounds were of $95 \%$ purity or better and used as received in solid form. 4-Acetamidophenol, carbamazepine, (+)-cis-diltiazem hydrochloride, norfluoxetine hydrochloride, ranitidine hydrochloride, and sulfamethoxazole were obtained from Sigma (St. Louis, MO, USA). Caffeine was obtained from Alfa Aesar (Ward Hill, MA, USA). Trimethoprim was obtained from Bufa (Uitgeest, Holland). Atorvastatin and lovastatin were obtained from Rugao (Shanghai, China). Levofloxacin was obtained from Zhejiang Wonderful Pharma \& Chemical (Zhejiang, China). Sertraline was obtained from Ranbaxy Laboratories (New Delhi, India). The internal standard (IS), ${ }^{13} \mathrm{C}$-caffeine was obtained from Isotec (Miamisburg, $\mathrm{OH}$, USA) and received as a $1.0-\mathrm{mg} / \mathrm{mL}$ solution in pure methanol $(\mathrm{MeOH})$.

Ultrapure water (MQ) was of $18.2 \mathrm{M} \Omega \mathrm{cm}$ purity and supplied by a Direct-Q 3 UV system (Millipore, Billerica, MA, USA). All organic solvents (acetonitrile (ACN), $\mathrm{MeOH}$, and isopropanol (IPA)) were Optima grade purity (Fisher Scientific, Pittsburgh, PA, USA) and 99\% pure formic acid (FA) was purchased from Acros Organics (Morris Plains, NJ, USA). Stock solutions of standards were made by weighing out approxi-

Table 1

Molecular structure and class designation of target pharmaceutical analytes

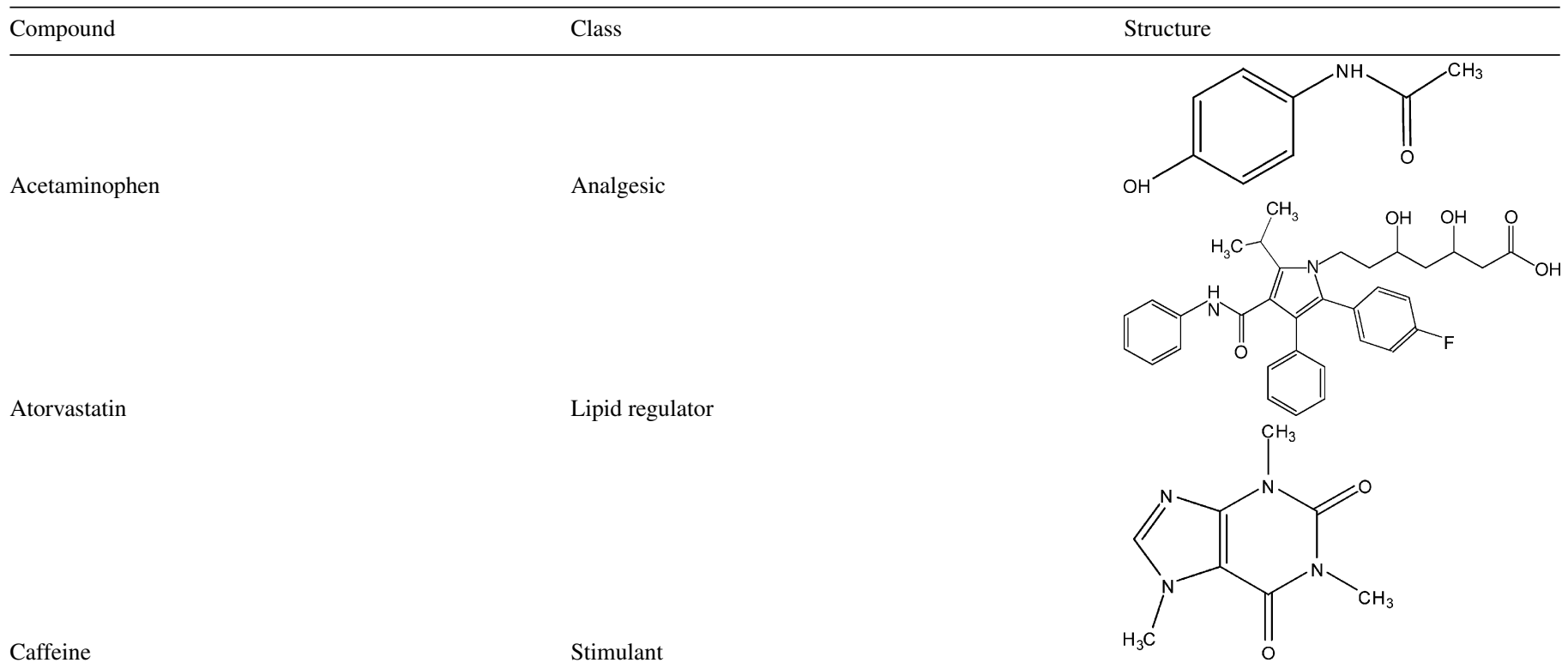


Table 1 (Continued)

\begin{tabular}{l} 
Compound \\
\hline Carbamazepine
\end{tabular}

Ciprofloxacin

\section{Diltiazem}

Fluoxetine

\section{Levofloxacin}

Lovastatin
Class

Antiepileptic

Antibiotic

Antihypertensive

Antidepressant

Antibiotic

Lipid regulator

Fluoxetine metabolite
Structure<smiles>NC(=O)n1c2ccccc2ccc2ccccc2n1C1CC1n1cc(C(=O)O)c(=O)c2cc(F)c(N3CCNCC3)cc21</smiles><smiles>COc1ccc([C@H]2Sc3ccccc3N(CCN(C)C)C(=O)[C@H]2OC(C)=O)cc1</smiles><smiles>CNCC[C@@H](Oc1ccc(C(F)(F)F)cc1)c1ccccc1</smiles><smiles>CCC(=O)CC1COn2cc(C(=O)O)c(=O)c3cc(F)c(N4CCN(C)CC4)c1c32</smiles><smiles>CCC(C)C(=O)OC1CC(C)C=CC=CC(C)[C@H]1CCC[C@H](O)CC</smiles><smiles>[NH]CC[C@@H](Oc1ccc(C(F)(F)F)cc1)c1ccccc1</smiles> 
Table 1 (Continued)

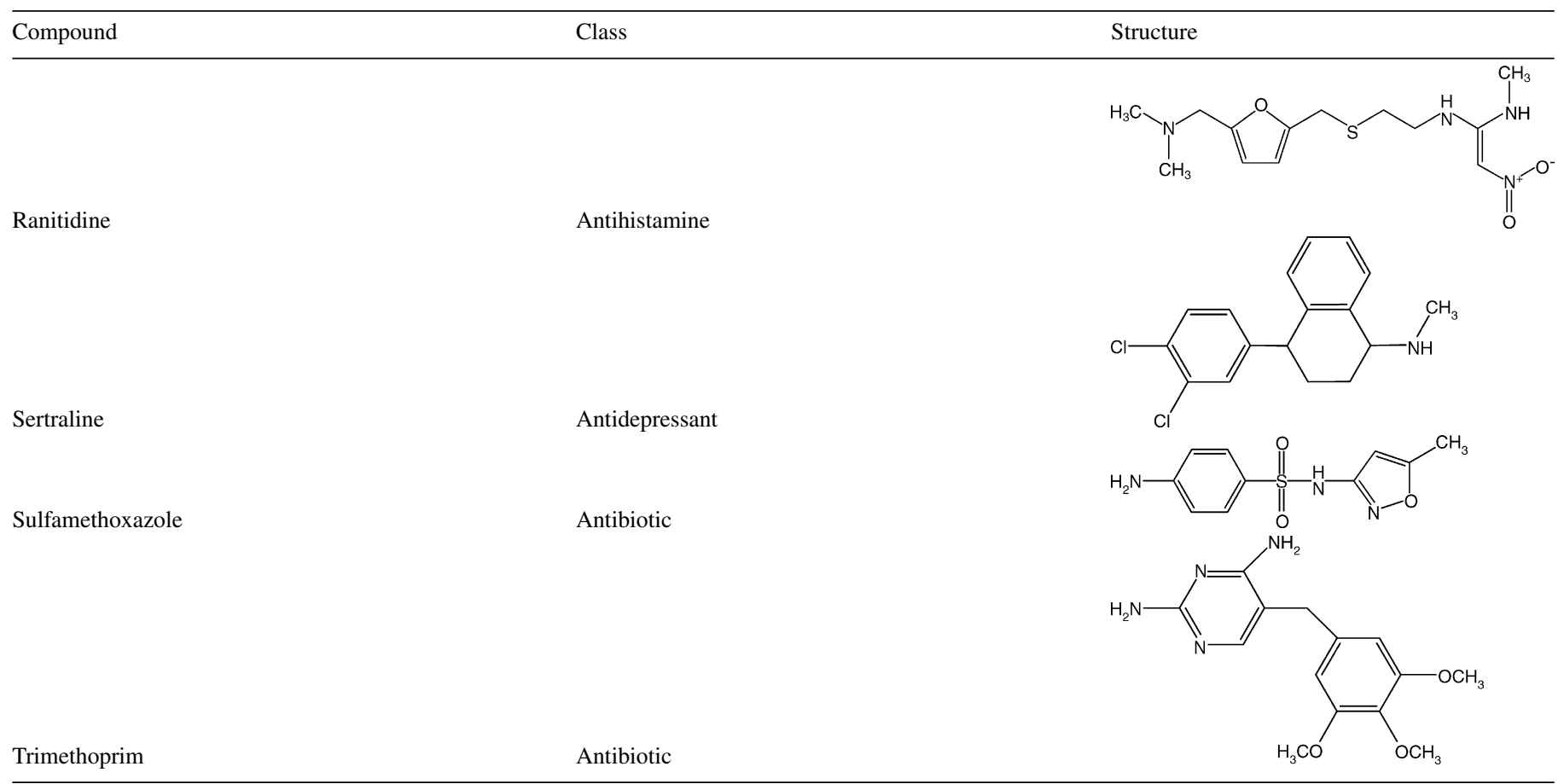

mately $100 \mathrm{mg}$ (three significant figures) solid standard of each drug and dissolving in 1.0 L of a 50:50 mix of MQ:ACN. The stock solution was subsequently $0.22 \mu \mathrm{m}$ filtered into an amber glass vial and stored at $4{ }^{\circ} \mathrm{C}$. Working solutions were prepared daily by dilution of stock solution into a mix of $85: 15$ MQ:ACN + 0.1\% FA. Stock solutions were kept no longer than 3 months and prepared fresh as needed for sample quantitation.

\subsection{Sample collection}

Surface water samples were collected from the Upper Tennessee River Basin 12-13 December 2006. Samples were collected as a composite of three vertical profiles from the river cross-section with $500 \mathrm{~mL}$ aliquots being collected equidistant from the right bank and left bank and from the center of the river. Aliquots were homogenized and $1.0 \mathrm{~L}$ was transferred to an amber HDPE bottle and placed on ice. Sample bottles were cleaned prior to sampling by triple rinsing with a 50:50 mix of MeOH:IPA and with MQ. Within $12 \mathrm{~h}$ samples were filtered through $47 \mathrm{~mm}, 0.77 \mu \mathrm{m}$ glass fiber filters conditioned with approximately $10 \mathrm{~mL}$ of a 50:50 mix of MeOH:IPA and $10 \mathrm{~mL}$ of MQ. Samples were additionally passed through $47 \mathrm{~mm}, 0.45 \mu \mathrm{m}$ membrane filters and then frozen at $-80^{\circ} \mathrm{C}$ until analysis.

\subsection{Sample preparation}

Samples were removed from the freezer and allowed to thaw to room temperature gradually $(\sim 16 \mathrm{~h})$. Bottles were homogenized and exactly $500 \mathrm{~mL}$ were transferred to a volumetric flask and amended with $50 \mu \mathrm{L}$ of a $1.0-\mathrm{mg} / \mathrm{L}$ solution of ${ }^{13} \mathrm{C}$ caffeine IS (final conc., $100 \mathrm{ng} / \mathrm{L}$ ). Analytes were concentrated using Waters (Milford, MA, USA) Oasis hydrophilic-lipophilic balanced (HLB), $60 \mathrm{mg}, 3 \mathrm{~mL}$ SPE cartridges in batches of 8 . Cartridges were mounted in a vacuum manifold fitted with polytetrafluoroethylene (PTFE) stopcock/needles. Each cartridge was conditioned with $3 \mathrm{~mL} \mathrm{MeOH}$ followed by $3 \mathrm{~mL} \mathrm{MQ}$. Samples were then drawn through the cartridges at a rate of approximately $10 \mathrm{~mL} / \mathrm{min}$ using PTFE transfer tubes. After sample loading was complete, sample flasks and transfer tubes were rinsed with $10 \mathrm{~mL}$ of MQ and the rinsate was passed through the SPE cartridge. A wash step of $3 \mathrm{~mL}$ of MQ was then passed through the SPE cartridges. Cartridges were then dried under vacuum for $15 \mathrm{~min}$.

Dried cartridges were transferred to a modified elution chamber consisting of a $16 \mathrm{~mm} \times 100 \mathrm{~mm}$ glass test tube with a LC sample vial inserted. Analytes were eluted using $500 \mu \mathrm{L}$ of $\mathrm{MeOH}$ followed by $500 \mu \mathrm{L}$ of acidified $\mathrm{MeOH}(0.5 \mathrm{M} \mathrm{FA})$. Each solvent was allowed to drip through the cartridge under gravity for $10 \mathrm{~min}$. Elution chambers were then centrifuged for $2 \mathrm{~min}$ to maximize analyte recovery. Sample vials were then evaporated to dryness under a gentle stream of nitrogen gas at room temperature. Dried sample vials were reconstituted with $500 \mu \mathrm{L}$ of sample diluent (85:15 MQ:ACN + 0.1\% FA), resulting in a 1000fold concentration of analytes. If instrumental analysis could not be completed immediately, sample vials were frozen at $-20^{\circ} \mathrm{C}$ until they could be analyzed, typically within $48 \mathrm{~h}$.

Quality control was monitored through field blanks, which consisted of two $500 \mathrm{~mL}$ aliquots of MQ being handled in the same manner as field samples. Additionally, method blanks and method spikes were processed without contact to the field sampling equipment. Method blanks consisted of MQ amended with the IS, and method spikes consisted of MQ spiked with either 50 or $100 \mathrm{ng} / \mathrm{L}$ of all analytes as well as the IS. One method blank and one method spike was analyzed to monitor for con- 
tamination and recovery of analytes from the sample preparation procedure.

\subsection{Chromatography and mass spectrometry}

Separation of compounds was carried out using a Waters Acquity ultra performance liquid chromatograph (UPLC ${ }^{\mathrm{TM}}$ ) fitted with a $50.0 \mathrm{~mm} \times 2.1 \mathrm{~mm}$, bridged-ethyl-siloxane/silica hybrid (BEH) Shield RP-C 18 column coupled with a 30.0-mm $\mathrm{C}_{18}$ guard column. Separation was achieved using a step-wise binary elution gradient consisting of 95:5 MQ:ACN +0.1\% $\mathrm{FA}$ as the aqueous phase (A) and $\mathrm{ACN}+0.1 \% \mathrm{FA}$ as the organic phase (B). The gradient was as follows: $5 \% \mathrm{~B}$ held for $0.70 \mathrm{~min}$, increased linearly to $30 \% \mathrm{~B}$ in $0.10 \mathrm{~min}$, then a gradual increase to $35 \% \mathrm{~B}$ over $1.00 \mathrm{~min}$, followed by an increase to $95 \% \mathrm{~B}$ in $0.1 \mathrm{~min}$ and held for $0.3 \mathrm{~min}$, finally a return to initial conditions of $5 \% \mathrm{~B}$ over $0.25 \mathrm{~min}$ and held for $0.80 \mathrm{~min}$ to allow for equilibration before the next injection. All flow was directed into the mass spectrometer. Additional parameters were as follows: flow rate, $0.400 \mathrm{~mL} / \mathrm{min}$; injection volume, $5 \mu \mathrm{L}$; column temperature, $40^{\circ} \mathrm{C}$; sample temperature, $25^{\circ} \mathrm{C}$.

The UPLC was interfaced to a Quattro Micro API triplequadrupole mass spectrometer (Micromass, Manchester, UK) with an electrospray ionization (ESI) source. The mass analyzer operated in positive ionization mode for all analytes and ESI source conditions were as follows: source temperature, $130{ }^{\circ} \mathrm{C}$; desolvation temperature, $400^{\circ} \mathrm{C}$; cone gas, $25 \mathrm{~L} / \mathrm{h}$; desolvation gas, $750 \mathrm{~L} / \mathrm{h}$; capillary voltage, $0.7 \mathrm{kV}$; multiplier, $650 \mathrm{~V}$. Nitrogen (99.995\% purity, Airgas) was used as the desolvation and nebulization gas, and ultra-pure argon (99.999\% purity, Airgas) was used as collision gas.

To determine the mass transitions of analytes, a $100-\mu \mathrm{g} / \mathrm{L}$ solution of each compound was infused directly into the mass spectrometer at $10 \mu \mathrm{L} / \mathrm{min}$. The flow path was modified with a T-mixer, which allowed LC mobile phase to be simultaneously infused with the sample solution. This allowed the analytes to reach the ESI source under similar conditions as those that would be encountered during a typical sample analysis. For all analytes the precursor ion corresponding to $\mathrm{m} / \mathrm{z}[\mathrm{M}+\mathrm{H}]^{+}$was determined to be the most dominant ion, except for lovastatin for which $\mathrm{m} / z[\mathrm{M}+\mathrm{Na}]^{+}$was the most dominant ion. The most abundant product ions were determined by adjusting the collision cell energy and pressure of argon in the collision cell. Table 2 reports the mass transitions, cone, and collision energies used for each of the analytes.

\subsection{Quantitation}

The standard addition method was used to quantify all analytes. Briefly, each reconstituted sample was separated into four equal aliquots $(125 \mu \mathrm{L})$. The order of the four vials was randomized in the sample plate to discourage any biases created from pipetting. Each vial was then amended with a $12.5-\mu \mathrm{L}$ aliquot of standard solution. Specifically, vial 1 was amended with sample diluent containing no analytes, vial 2 was amended with a 10.0$\mu \mathrm{g} / \mathrm{L}$ solution of each of the analytes, vial 3 with a $100.0-\mu \mathrm{g} / \mathrm{L}$ solution, and vial 4 with a $1000.0-\mu \mathrm{g} / \mathrm{L}$ solution. Samples were injected in triplicate and in increasing concentration order with two blanks injected between samples to minimize carryover. All peak data was acquired using Mass Lynx software (Version 4.1, Waters Corp.).

Analytes were detected using multiple reaction monitoring (MRM) mode for the appropriate mass transitions. MS method parameters include: inter-channel delay, $30 \mathrm{~ms}$; inter-scan delay, $30 \mathrm{~ms}$; repeats, 1 . The dwell time varied for each analyte, depending on the number of analytes within a given time frame, to allow for an adequate number of scans $(\geq 12)$ to resolve the peak. Mass spectrometry dwell times were either $50 \mathrm{~ms}$ (diltiazem, norfluoxetine, fluoxetine, sertraline, sulfamethoxazole), $100 \mathrm{~ms}$ (acetaminophen, trimethoprim, caffeine, ${ }^{13} \mathrm{C}_{3}$-caffeine, levofloxacin, ciprofloxacin, lovastatin), or $200 \mathrm{~ms}$ (ranitidine, carbamazepine, atorvastatin).

Table 2

Mass spectrometric parameters for detection of pharmaceutical compounds

\begin{tabular}{|c|c|c|c|c|c|}
\hline Time (min) & Analyte & Precursor ion $(\mathrm{m} / \mathrm{z})$ & Product ion $(\mathrm{m} / \mathrm{z})$ & Cone $(\mathrm{V})$ & Collision (V) \\
\hline 0.42 & Ranitidine & 315.15 & 176.10 & 25 & 17 \\
\hline 0.79 & Acetaminophen & 152.00 & 109.70 & 30 & 16 \\
\hline 0.80 & Trimethoprim & 291.15 & 230.30 & 38 & 24 \\
\hline 1.05 & Caffeine & 195.00 & 138.00 & 34 & 20 \\
\hline 1.05 & ${ }^{13} \mathrm{C}$-Caffeine & 198.00 & 139.90 & 35 & 19 \\
\hline 1.11 & Levofloxacin & 362.20 & 318.30 & 32 & 18 \\
\hline 1.28 & Ciprofloxacin & 332.20 & 288.30 & 34 & 16 \\
\hline 1.36 & Diltiazem & 415.20 & 178.10 & 32 & 24 \\
\hline 1.48 & Norfluoxetine & 296.10 & 133.90 & 14 & 6 \\
\hline 1.52 & Fluoxetine & 310.10 & 148.00 & 19 & 9 \\
\hline 1.55 & Sertraline & 306.10 & 275.20 & 17 & 13 \\
\hline 1.54 & Sulfamethoxazole & 254.10 & 155.90 & 26 & 16 \\
\hline 1.87 & Carbamazepine & 237.10 & 194.20 & 30 & 19 \\
\hline 2.47 & Atorvastatin & 559.20 & 440.35 & 34 & 22 \\
\hline 2.58 & Lovastatin & $427.10^{\mathrm{a}}$ & 325.30 & 42 & 22 \\
\hline
\end{tabular}

Retention time and appropriate mass transition were used to positively identify compounds by monitoring for the protonated form of the molecule $[\mathrm{M}+\mathrm{H}]^{+}$unless otherwise noted. ${ }^{13} \mathrm{C}$-Caffeine-internal standard.

${ }^{\mathrm{a}}[\mathrm{M}+\mathrm{Na}]^{+}$. 
All peak integration and analysis was performed using QuanLynx (Version 4.1, Waters Corp.). Peaks were smoothed using mean smoothing with one iteration. A response factor ((sample peak area) $\times($ IS conc./IS area)) was calculated for each peak using the IS to correct for small variations. Response factors were used to generate a linear regression $(y=m x+b)$ with $1 / x$ weighting and excluding the origin. Analyte concentrations were determined by taking the absolute value of the intercept $(b)$ divided by the slope $(m)$ and applying the 1000-fold concentration factor to finish with units of $\mathrm{ng} / \mathrm{L}$.

\section{Results and discussion}

\subsection{Chromatographic method}

A variety of solvents were tested during the development of the elution gradient. These included $\mathrm{MeOH}$ and $\mathrm{ACN}$ for the $\mathrm{B}$ channel, and $0.5 \mathrm{mM}$ ammonium acetate, pure MQ, and combinations of organic solvent + MQ for the A channel. It was determined that the combination of 95:5 MQ:ACN (A channel) and ACN (B channel), each with $0.1 \%$ FA added, provided the most efficient separation and best peak shape for all analytes in the method in a favorably short run time.

Trials indicated that $\mathrm{MeOH}$ was not a strong enough elution solvent due to extended retention times of the most nonpolar compounds, and peak broadening or overall poor peak shape for the most polar compounds. Having 5\% ACN in the aqueous channel helped to reduce the influence of pressure spikes on column during the rapid, step-wise changes in the elution gradient. Formic acid improved peak shape and area by providing a source of protons to aid in the overall ionization efficiency of the source.

The goal of the chromatographic separation was to resolve peaks while having an adequate number of scans across each peak ( $\geq 12$ scans) in the shortest time frame possible, while retaining acceptable peak shape. Due to the variety of physicochemical properties displayed by the analytes, including large differences in polarities, it was determined a step-wise binary elution gradient would be the most efficient. The most polar molecules (ranitidine, acetaminophen, trimethoprim, caffeine) were eluted during the initial gradient step (5\% B). Next, the moderately nonpolar molecules (ciprofloxacin, levofloxacin, diltiazem, norfluoxetine, fluoxetine, sertraline, sulfamethoxazole, and carbamazepine) were eluted during the second step (30-35\% B). Finally, the most nonpolar molecules (atorvastatin and lovastatin) were eluted during the third step (90\% B). Tests were done with a purely linear elution gradient, but this resulted in run times of up to $12 \mathrm{~min}$. By quickly ramping $(0.1 \mathrm{~min})$ the change in B channel between gradient steps, all analytes were adequately separated and eluted to the detector in $3.25 \mathrm{~min}$, including a return to initial conditions and a 0.8 -min column equilibration step before the next sample was injected.

\subsection{Mass spectrometry}

All precursor ions determined here were essentially identical to those reported in other LC-MS/MS methods for the compounds acetaminophen, atorvastatin, caffeine, carba- mazepine, ciprofloxacin, diltiazem, fluoxetine, levofloxacin, norfluoxetine, ranitidine, sertraline, sulfamethoxazole, and trimethoprim [15,17,18,21,23,24,29,30]. Product ions varied among other published methods but similar complete mass transitions were reported for each drug including acetaminophen [17,21,24,26,29], atorvastatin [16], caffeine [17,18,26,29], carbamazepine [17,21-24,26,29,30], ciprofloxacin [22], diltiazem [18,29,30], fluoxetine [29,31], levofloxacin [26], norfluoxetine [29], sertraline [26,29], sulfamethoxazole [17,18,26,29,30], and trimethoprim $[15,21,24,30]$. Several studies had similar precursor ions, but dissimilar product ions than those used in this method, including sulfamethoxazole [15,24], fluoxetine [17], trimethoprim [17,18,23], and ciprofloxacin [30]. Lovastatin was found in the literature to have a different mass transition $(\mathrm{m} / \mathrm{z}$ $436>285$ ) than reported for this method [16]. To our knowledge, the mass transition used for the determination of lovastatin has not been published previously.

\subsection{Solid-phase extraction-sample loading}

Oasis HLB SPE cartridges are commonly used for analysis of pharmaceuticals in environmental matrices [16-18,20-24,32,33]. Few studies indicate the reasoning for utilizing one mass of HLB sorbent versus another. Typically the concern is whether the given sorbent mass has the capacity to effectively retain the analytes of choice without overloading the active sites. A simple breakthrough study was conducted to determine analyte retention when loading onto a 60-mg Oasis HLB cartridge. Two $500 \mathrm{~mL}$ aliquots of MQ, spiked at $100 \mu \mathrm{g} / \mathrm{L}$ for all analytes, were loaded on pre-conditioned $60 \mathrm{mg}$ Oasis HLB cartridges at $10 \mathrm{~mL} / \mathrm{min}$. All filtrate for each cartridge was collected separately and breakthrough was quantified by comparing the signal of a sample of the spiked water prior to cartridge loading to a sample of the collected filtrate. With the exception of acetaminophen, ranitidine, and caffeine, all drugs displayed less than $1 \%$ breakthrough. Acetaminophen displayed a mean breakthrough of $44.7 \%$, ranitidine $2.09 \%$, and caffeine $1.69 \%$. It is assumed that these analytes were less adequately retained due to their high polarity relative to the other analytes.

The second test of cartridge efficacy was based on the $\mathrm{pH}$ of the sample (data not shown). Duplicate MQ spikes (100 ng/L) were prepared at $\mathrm{pH} 2,7$, and 10 using concentrated sulfuric acid or sodium hydroxide. The recoveries indicated that at $\mathrm{pH} 2$ ranitidine was essentially not recovered, most likely due to molecular ionization at this $\mathrm{pH}$ and subsequent breakthrough of the SPE sorbent. Other drugs, including ciprofloxacin, diltiazem, sertraline, fluoxetine, and norfluoxetine displayed higher average recoveries at $\mathrm{pH} 2$ than the other $\mathrm{pHs}$. At $\mathrm{pH} 10$ sulfamethoxazole, acetaminophen, and lovastatin displayed low recoveries, most likely because of poor retention due to molecular ionization or inadequate elution. Further, at pH 10 norfluoxetine, fluoxetine, sertraline, and diltiazem displayed reduced recoveries as compared to the other $\mathrm{pHs}$. At $\mathrm{pH} 7$ no compounds displayed dramatic adverse effects and, in many instances, recoveries were as good or better than the other pHs. For this reason, pH 7 was chosen for sample preparation and environmental samples were not amended with acids or bases. 
Therefore, it was determined that $60 \mathrm{mg}$ HLB cartridges provided adequate retention at neutral $\mathrm{pH}$ for the majority of analytes. Acetaminophen displayed a relatively large breakthrough $(\sim 45 \%)$ but in order to achieve the most optimum retention for a broad spectrum of analytes, it is necessary to make decisions that might adversely affect one or more of the compounds. In this case, acetaminophen is commonly found in the environment in relatively high concentrations $[7,24]$ so a low recovery is not seen as an unjustifiable hindrance to quantitation. Further verification for the use of $60 \mathrm{mg}$ HLB cartridges can be seen in a number of other studies which utilize this cartridge and have achieved adequate recoveries (generally 60-100\%) [22-24,32]. Additionally, $60 \mathrm{mg}$ HLB cartridges are the least costly (two- to fourfold less) of the Oasis HLB syringe-type SPE cartridges.

\subsection{Solid-phase extraction-sample elution}

As previously described, it was observed that the majority of analytes were fully retained on the HLB sorbent and thus, could potentially be completely eluted. A variety of organic solvents were tested for elution efficiency including: MTBE, MeOH, IPA, $\mathrm{ACN}$, hexane, and ethanol. It was determined that $\mathrm{MeOH}$ was the most adequate for eluting analytes using minimal elution volumes. This elution process is similar to the one utilized by Lam et al. [26] for a similar array of analytes.

The use of a small volume of elution solvent allowed the eluent to be collected directly into a LC sample vial. This helped eliminate the error associated with passing the eluent through the PTFE stopcock/needle unit and reducing the number of glassware and sample transfers during elution, evaporation, and reconstitution steps. It could be argued that the low recovery for a number of analytes ( $<70 \%$ for: ranitidine, acetaminophen, sulfamethoxazole, atorvastatin, and lovastatin) was due to inadequate elution solvent volume to remove all analytes. This is most likely true, however it is not seen as a hindrance due to the acceptable reproducibility (RSDs $\leq 16 \%$ ) for recovery of analytes across multiple days $(n=3)$. Further, the amount of elution solvent employed in this method is four- to eightfold less than in other published methods using the same SPE cartridge in a similar context [22-24].

\subsection{SPE recovery and matrix effect}

Analyte recovery was determined using assessment techniques similar to those outlined by Matuszewski et al. [34] for dealing with complex matrices. Surface water was collected from the Tennessee River at a site upstream of any known sewage treatment plant discharge points to reduce the interference of ambient pharmaceutical concentrations. On each of 3 days, $4 \times 1000 \mathrm{~mL}$ samples were collected from the river and filtered according to experimental procedure. Filtered samples were homogenized in a 4000-mL amber glass jug and separated into $8 \times 500 \mathrm{~mL}$ volumetric flasks. Four flasks were amended with $50 \mu \mathrm{L}$ of a $1.0-\mathrm{mg} / \mathrm{L}$ mixture $(0.1 \mu \mathrm{g} / \mathrm{L}$ final conc.) of all analytes (Set 1) and the remaining four flasks were amended with $50 \mu \mathrm{L}$ of pure sample diluent (Set 2). All eight samples were taken through the sample preparation procedure except for
Set 2 samples, which were reconstituted with $500 \mu \mathrm{L}$ of a 100$\mu \mathrm{g} / \mathrm{L}$ mixture of all analytes. Sets 1 and 2 samples were analyzed and recovery was determined for each analyte using peak areas with the following equation:

Recovery $(\%)=\left(\frac{\text { Set } 1 \text { Area }}{\text { Mean Set } 2 \text { Area }}\right) \times 100$

Recovery was assessed for reproducibility each day (intraday reproducibility) and across days (interday reproducibility). The overall recovery of the method as reported in Table 3 is the interday reproducibility, as this is seen as a better qualifier of the robustness of the method. To determine interday reproducibility, the entire set of recoveries $(n=12)$ was averaged for each drug and $\%$ RSD calculated.

The majority of the molecules that elute in the middle of the LC gradient (trimethoprim, caffeine, levofloxacin, ciprofloxacin, diltiazem, norfluoxetine, fluoxetine, sertraline, carbamazepine) display very good recoveries (range, 71.5$104.9 \%$ ). Acetaminophen was observed to suffer a low recovery $(52.8 \%)$ but this can be explained by the significant breakthrough $(\sim 45 \%)$ of the SPE sorbent. The remaining molecules displaying low recovery (ranitidine, sulfamethoxazole, atorvastatin, lovastatin) are most likely due to incomplete desorption from the SPE sorbent during the elution step.

Matrix effect was determined parallel to the recovery study. In addition to Sets 1 and 2 described above, Set 3 consisted of a $100-\mu \mathrm{g} / \mathrm{L}$ solution of all analytes in neat sample diluent. To assess percent matrix effect for each analyte, peak areas were used with the following equation:

Matrix Effect $(\%)=\left(\left(\frac{\text { Mean Set } 2 \text { Area }}{\text { Mean Set } 3 \text { Area }}\right)-1\right) \times 100$

This equation is similar to that described by Matuszewski et al. [34], but has been slightly modified as stated by Clodfelter [35]. A negative (-) value indicates matrix suppression and a positive (+) value indicates matrix enhancement. It was observed that all analytes suffered from matrix suppression (range, -8.7 to $-79.3 \%$; mean, $-35.0 \%$ ), most likely from analyte competition for protonation at the ESI source with constituent matrix components. Table 3 presents the instrumental limitations and method limitations, recovery, and matrix effect.

\subsection{Calibration and quantitation}

Numerous studies have been performed explicitly to deal with matrix effects when quantifying compounds in environmental samples using LC-MS/MS [36-40]. Proposed methods involve extensive sample clean-up during sample preparation, decreased flow or flow splitting of mobile phase into the MS source, and dilution of samples prior to injection. Each of these methods typically results in a reduction in matrix effect but, consequently, a decrease in analyte signal at the detector or reduced SPE recovery may occur. Isotopically labeled ISs are also frequently utilized but unless there is an IS congruent to each analyte in the method, the researcher is taking a potentially invalid assumption that the matrix is affecting the analyte in an identical way to the ISs. Further, com- 
Table 3

Instrumental limitations and method parameters of efficacy

\begin{tabular}{|c|c|c|c|c|c|}
\hline \multirow[t]{2}{*}{ Analyte } & \multicolumn{2}{|c|}{ Instrumental } & \multicolumn{3}{|l|}{ Method } \\
\hline & LOD (pg) & LOQ (pg) & MDL (ng/L) & Recovery (\%RSD) & Matrix effect (\%) \\
\hline Ranitidine & 0.271 & 0.900 & 1.77 & $64.3(8.7)$ & -28.7 \\
\hline Acetaminophen & 1.27 & 4.22 & 2.06 & $52.8(14.2)$ & -25.1 \\
\hline Trimethoprim & 0.320 & 1.07 & 2.26 & $96.7(4.2)$ & -8.7 \\
\hline Caffeine & 0.990 & 3.30 & 17.7 & $95.3(2.9)$ & -14.6 \\
\hline Levofloxacin & 0.481 & 1.61 & 5.95 & $104.9(9.5)$ & -11.3 \\
\hline Ciprofloxacin & 0.550 & 1.84 & 4.51 & $93.6(7.5)$ & -49.5 \\
\hline Diltiazem & 1.04 & 3.45 & 1.25 & $88.3(4.0)$ & -37.0 \\
\hline Norfluoxetine & 0.0452 & 0.151 & 1.84 & $71.5(4.7)$ & -42.4 \\
\hline Fluoxetine & 0.815 & 2.72 & 3.29 & $80.1(3.6)$ & -38.0 \\
\hline Sertraline & 0.472 & 1.57 & 1.92 & $80.6(3.0)$ & -47.3 \\
\hline Sulfamethoxazole & 0.295 & 0.980 & 2.97 & $54.9(10.3)$ & -41.0 \\
\hline Carbamazepine & 0.317 & 1.06 & 2.85 & $99.3(2.2)$ & -18.9 \\
\hline Atorvastatin & 0.620 & 2.07 & 2.24 & $42.0(11.3)$ & -47.8 \\
\hline Lovastatin & 0.505 & 1.69 & 6.53 & $67.0(16.2)$ & -79.3 \\
\hline
\end{tabular}

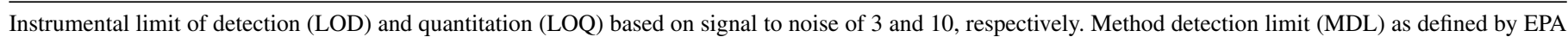
[40] $(n=10)$; recovery and matrix effect determined similar to Matuszewski et al. [34]. RSD: relative standard deviation; pg: picograms of analyte on-column.

mercially available ISs do not exist for all commonly used pharmaceuticals, including a number of analytes found in this study and, when available, can be considerably cost intensive.

Standard addition is widely viewed as the only accurate alternative to comprehensive internal standardization for quantifying analytes in complex matrices using LC-MS/MS [36,37]. Standard addition is seen as being time consuming and laborious, but it is able to compensate for matrix interference given a sampling paradigm covering a broad area with wide variation in matrix components and a lack of available isotopically labeled standards.

Due to the relatively high degree of matrix suppression (mean, -35.0\%), large sampling area (Upper Tennessee River Basin), and objective to keep method costs to a minimum, standard addition was chosen to quantify all compounds in the method. Although standard addition can be performed with only one spiked sample [37], our method utilized a four point linear regression. All analytes displayed excellent linearity $\left(r^{2} \geq 0.995\right)$ when analyzed as neat standard solutions. Further, each sample was amended with an IS to correct for small variations in pipetting and sample injection volume. Response factors were only calculated within individual samples because the individual matrix of each sample influenced the IS differently.

Finally, all concentrations were corrected for recovery to determine final concentrations by dividing the calculated concentration by the percent recovery. The majority of methods in the peer reviewed literature for determination of pharmaceuticals in environmental matrices do not indicate whether final concentrations were corrected for recovery or not. Of the methods cited in this paper, only Lee et al. [25] indicate that they did not correct for recovery. We conclude that the acceptably low variation in recovery $(<16 \%, n=12$ across 3 days) allowed it to be used as a correction factor in the final determination of pharmaceutical concentrations in environmental sample analysis.

\subsection{Analytical limitations}

Instrumental limits of detection and quantitation (LOD and LOQ) were defined as a signal to noise ratio of 3 and 10, respectively. LODs and LOQs were calculated by analyzing a low concentration standard solution $(0.5 \mu \mathrm{g} / \mathrm{L}$ or $0.1 \mu \mathrm{g} / \mathrm{L})$, using software assisted techniques to calculate a signal to noise ratio (Peak to Peak algorithm in Mass Lynx), and extrapolating to the defined ratios for LOD and LOQ. For each analyte the lowest concentration that resulted in a definable peak was used. Each response was corrected by subtracting the signal-to-noise ratio of any peaks present in blanks from the signal-to-noise ratio of the standard solution. Finally, the units were normalized from $\mu \mathrm{g} / \mathrm{L}$ to $\mathrm{pg} / \mathrm{injection}$ for comparison with other methods. LOD (range, 0.0452-1.27 pg/inj.; mean, 0.571 pg/inj.) and LOQ (range, 0.151-4.22 pg/inj.; mean, $1.90 \mathrm{pg} / \mathrm{inj}$.) values were as low or lower when compared to other LC-MS/MS methods for pharmaceuticals reporting pg/injection [17,20-22,24].

Method detection limits (MDL) are the most useful indicators of the overall utility of an analytical method. The EPA defines an MDL as the minimum concentration of a substance that can be measured and reported with $99 \%$ confidence that the analyte concentration is greater than 0 [41]. In the present study 10 replicate samples $(500 \mathrm{~mL})$ of Tennessee River water were spiked at $10 \mathrm{ng} / \mathrm{L}$ and 10 were unspiked in order to subtract background levels of analytes. Each spike and blank was processed, analyzed, and quantified according to the present method. Mean concentration of each analyte was calculated for the 10 replicate blanks and subtracted from each of the spiked samples. Next, the standard deviation $(S)$ was calculated for the blank subtracted spiked samples and that value was used to determine the MDL using the equation:

$\mathrm{MDL}=T_{(n-1, \alpha=0.01)} \times S$

where $T=2.821$ for 9 degrees of freedom at $\alpha=0.01$. MDL values (range, $1.25-17.7 \mathrm{ng} / \mathrm{L}$; mean, $4.08 \mathrm{ng} / \mathrm{L}$ ) were also in the same order of magnitude (low $\mathrm{ng} / \mathrm{L}$ ) as other similar methods 
$[18,21,24,25]$ and adequately low for analysis of surface water samples.

\subsection{Environmental assessment and method application}

The method was applied to surface water samples collected from the Upper Tennessee River located in east Tennessee. The sampling paradigm involved collecting surface water samples upstream and downstream of major sewage treatment plants that discharge directly into the Tennessee River between Knoxville, $\mathrm{TN}$ and Chattanooga, TN. Table 4 presents the raw values as quantified using the method.

Seven of the 14 analytes were detected (detection indicating a concentration $\geq$ MDL) in at least one sample. Those not detected include ciprofloxacin, diltiazem, fluoxetine, levofloxacin, norfluoxetine, ranitidine, and sertraline (Table 4). Caffeine, carbamazepine, and sulfamethoxazole were the most frequently detected pharmaceuticals with concentrations being detected in all samples. Trimethoprim was detected in 3 out of 10 samples. The remaining drugs (acetaminophen, atorvastatin, and lovastatin) were detected in 1 out of 10 samples. To our knowledge, the detection of lovastatin $(18.3 \mathrm{ng} / \mathrm{L})$ represents the first time this compound has been detected in surface water [16].

Caffeine displayed the highest mean reported concentration (mean of all samples \pm standard error, $28.9 \pm 1.39 \mathrm{ng} / \mathrm{L}$ ) as well as the single highest concentration $(38.8 \mathrm{ng} / \mathrm{L})$. This is similar to other studies analyzing for caffeine and is most likely due to the non-prescription status of caffeine and its inclusion in many common consumable products $[18,20]$. Sulfamethoxazole had the second highest mean concentration $(7.90 \pm 0.32 \mathrm{ng} / \mathrm{L})$, carbamazepine was third $(4.62 \pm 0.16 \mathrm{ng} / \mathrm{L})$, and trimethoprim was fourth $(4.09 \pm 1.20 \mathrm{ng} / \mathrm{L})$. Sulfamethoxazole, trimethoprim, and

Table 4

Pharmaceutical concentrations (ng/L) in Tennessee River surface water collected upstream (US) and downstream (DS) of five sewage treatment plants

\begin{tabular}{|c|c|c|c|c|c|c|c|}
\hline SITE & AMN & CAF & TMP & SMZ & $\mathrm{CAB}$ & ATV & LOV \\
\hline \multicolumn{8}{|l|}{1} \\
\hline US & - & 31.2 & 7.00 & 6.96 & 4.58 & - & 18.3 \\
\hline DS & - & 29.1 & - & 7.93 & 5.62 & - & - \\
\hline \multicolumn{8}{|l|}{2} \\
\hline US & - & 23.6 & - & 8.03 & 4.30 & - & - \\
\hline DS & - & 23.2 & - & 7.92 & 4.41 & - & - \\
\hline \multicolumn{8}{|l|}{3} \\
\hline US & 2.24 & 26.4 & - & 9.51 & 4.09 & 10.3 & - \\
\hline DS & - & 26.5 & - & 8.75 & 4.34 & - & - \\
\hline \multicolumn{8}{|l|}{4} \\
\hline US & - & 38.8 & - & 5.81 & 4.03 & - & - \\
\hline DS & - & 32.3 & - & 7.47 & 4.92 & - & - \\
\hline \multicolumn{8}{|l|}{5} \\
\hline US & - & 30.4 & 2.41 & 9.03 & 4.57 & - & - \\
\hline DS & - & 27.5 & 2.85 & 7.61 & 5.38 & - & - \\
\hline
\end{tabular}

Only those drugs with concentrations above their respective MDL are reported. No detection is reported as (-). AMN, acetaminophen; CAF, caffeine; TMP, trimethoprim; SMZ, sulfamethoxazole; $\mathrm{CAB}$, carbamazepine; ATV, atorvastatin; LOV, lovastatin. carbamazepine have all been reported to be commonly detected in STP impacted surface waters $[17,18,20,21]$. The remaining drugs did not have sufficient detections to calculate means and standard errors.

Field blanks displayed no detectable concentrations of pharmaceuticals above their respective MDLs. The only exception was atorvastatin, which displayed detection above the MDL in the field blank. This was most likely an artifact and not indicative of carryover. No consecutive samples displayed detections of atorvastatin and the site where the field blank was prepared also did not display detection of atorvastatin. This indicates that no carryover from site to site occurred. In addition, on-going field sampling has not indicated detection of atorvastatin in any field blanks (data not shown). Method blanks were all below MDL for every compound in the method. Method spikes were within $20 \%$ of the reported recoveries indicating the acceptable stability of freezing the samples at $-80^{\circ} \mathrm{C}$ between filtration and SPE.

The sampling effort and environmental concentrations reported here are parts of a larger field study of pharmaceuticals in the Upper Tennessee River Basin. The purpose of the full study is to investigate spatial and temporal variations in pharmaceutical concentrations and relate those to an indicator of aquatic invertebrate community health. The data represented here have not been statistically tested for differences in upstream versus downstream sampling sites as this will be performed at a later date with the complete dataset. The purpose of the present study was to describe and evaluate the analytical method and apply it to an initial sampling effort to determine if the method was reliable and reproducible under field conditions.

\section{Conclusions}

The LC-MS/MS method presented is rapid, relatively simple, and minimally cost intensive as compared to similar methods. The efficiency of the LC instrumentation employed allowed for significant reductions in sample analysis time and volume of mobile phase employed. Also, the use of smaller SPE sorbent masses with minimal elution volume, yet adequate recovery and acceptable variation, resulted in decreased costs associated with sample preparation. Further, the utility of the standard addition method to accurately quantify compounds in variable, complex sample matrices is cost favorable when compared to expensive, and often unavailable, isotopically labeled standards. The method has been validated through quality assurance/quality control assessment and applied to a collection of surface water samples. Levels of detected compounds are well within reason (low ng/L) as compared to other studies in the peer reviewed literature. Our results indicate that detectable levels of at least seven commonly used prescription and nonprescription pharmaceuticals are present in the Upper Tennessee River. Future sampling efforts will focus on elucidating spatial and temporal variations in pharmaceutical concentrations as well as potential toxicological effects on aquatic organisms. 


\section{Acknowledgments}

We would like to thank those who helped with problem solving during the development of the method: Kevin Chambliss (Baylor University), Chris Metcalfe (Trent University), and Cora Young (University of Toronto). We would also like to thank Keith Solomon (University of Guelph) for supplying some of the pharmaceuticals used in the method. Further, we would like to thank the National Science Foundation for funding this research and the Waters Corporation for supplying additional SPE materials.

\section{References}

[1] B. Halling-Sorensen, S.N. Nielsen, P.F. Lanzky, F. Ingerslev, H.C. Holten Lutzholft, S.E. Jorgensen, Chemosphere 36 (1998) 357.

[2] C.G. Daughton, T.A. Ternes, Environ. Health Perspect. 107 (1999) 907.

[3] O.A.H. Jones, N. Voulvoulis, J.N. Lester, Environ. Sci. Technol. 22 (2001) 1383.

[4] T. Heberer, Toxicol. Lett. 131 (2002) 5.

[5] K. Fent, A.A. Weston, D. Caminada, Aquat. Toxicol. 76 (2006) 122.

[6] C.G. Daughton, in: C.G. Daughton, T.L. Jones-Lepp (Eds.), Pharmaceuticals and Personal Care Products in the Environment: Scientific and Regulatory Issues, American Chemical Society, Washington, D.C., 2001, p. 2.

[7] D.W. Kolpin, E.T. Furlong, M.T. Meyer, E.M. Thurman, S.D. Zaugg, L.B. Barber, H. Buxton, Environ. Sci. Technol. 36 (2002) 1202.

[8] D.W. Kolpin, M. Skopec, M.T. Meyer, E.T. Furlong, S.D. Zaugg, Sci. Total Environ. 328 (2004) 119.

[9] G.R. Boyd, H. Reemtsma, D.A. Grimm, S. Mitra, Sci. Total Environ. 311 (2003) 135.

[10] S.A. Snyder, K.L. Kelly, A.H. Grange, G.W. Sovocool, E.M. Snyder, J.P. Giesy, in: C.G. Daughton, T.L. Jones-Lepp (Eds.), Pharmaceuticals and Personal Care Products in the Environment: Scientific and Regulatory Issues, American Chemical Society, Washington, D.C., 2001, p. 116.

[11] Kaiser Family Foundation, State Health Facts, 2007, http://www. statehealthfacts.org.

[12] T.A. Ternes, Trends Anal. Chem. 20 (2001) 419.

[13] M. Petrovic, M.D. Hernando, M. Silvia Diaz-Cruz, D. Barcelo, J. Chromatogr. A 1067 (2005) 1.

[14] S.D. Richardson, T.A. Ternes, Anal. Chem. 77 (2005) 3807.
[15] M.J. Hilton, K.V. Thomas, J. Chromatogr. A 1015 (2003) 129.

[16] X. Miao, C.D. Metcalfe, J. Chromatogr. A 998 (2003) 133.

[17] B.J. Vanderford, R.A. Pearson, D.J. Rexing, S.A. Snyder, Anal. Chem. 75 (2003) 6265.

[18] J.D. Cahill, E.T. Furlong, M.R. Burkhardt, D.W. Kolpin, L.G. Anderson, J. Chromatogr. A 1041 (2004) 171.

[19] M.D. Hernando, M. Petrovic, A.R. Fernandez-Alba, D. Barcelo, J. Chromatogr. A 1046 (2004) 133.

[20] R.A. Trenholm, B.J. Vanderford, J.C. Holady, D.J. Rexing, S.A. Snyder, Chemosphere 65 (2006) 1990.

[21] M.J. Gomez, M. Petrovic, A.R. Fernandez-Alba, D. Barcelo, J. Chromatogr. A 1114 (2006) 224.

[22] N.M. Vieno, T. Tuhkanen, L. Kronberg, J. Chromatogr. A 1134 (2006) 101.

[23] C. Hao, L. Lissemore, B. Nguyen, S. Kleywegt, P. Yang, K. Solomon, Anal. Bioanal. Chem. 384 (2006) 505.

[24] M. Gros, M. Petrovic, D. Barcelo, Talanta 70 (2006) 678.

[25] H. Lee, K. Sarafin, T.E. Peart, J. Chromatogr. A 1148 (2007) 158.

[26] M.W. Lam, C.J. Young, R.A. Brain, D.J. Johnson, M.A. Hanson, C.J. Wilson, S.M. Richards, K.R. Solomon, S.A. Mabury, Environ. Toxicol. Chem. 23 (2004) 1431.

[27] S.M. Richards, C.J. Wilson, D.J. Johnson, D.M. Castle, M.W. Lam, S.A. Mabury, P.K. Sibley, K.R. Solomon, Environ. Toxicol. Chem. 23 (2004) 1035.

[28] RxList, Top 200 Dispensed, http://www.rxlist.com.

[29] A.J. Ramirez, M.A. Mottaleb, B.W. Brooks, C.K. Chambliss, Anal. Chem. 79 (2007) 3155.

[30] R. Kasprzyk-Hordern, R.M. Dinsdale, A.J. Guwy, J. Chromatogr. A 1161 (2007) 132.

[31] T. Vasskog, U. Berger, P.J. Samuelsen, R. Kallenborn, E. Jensen, J. Chromatogr. A 1115 (2006) 187.

[32] M. Petrovic, M. Gros, D. Barcelo, J. Chromatogr. A 1124 (2006) 68.

[33] M. Huerta-Fontela, M.T. Galceran, F. Ventura, Anal. Chem. 79 (2007) 3821.

[34] B.K. Matuszewski, M.L. Constanzer, C.M. Chavez-Eng, Anal. Chem. 75 (2003) 3019.

[35] K. Clodfelter, Waters Corp., Milford, MA, August 2007, personal communication.

[36] M. Stuber, T. Reemtsma, Anal. Bioanal. Chem. 378 (2004) 910.

[37] S. Ito, K. Tsukada, J. Chromatogr. A 943 (2001) 39.

[38] T. Benijts, R. Dams, W. Lambert, A.D. Leenheer, J. Chromatogr. A 1029 (2004) 153.

[39] A. Kloepfer, J.B. Quintana, T. Reemtsma, J. Chromatogr. A 1067 (2005) 153.

[40] J.C. Van De Steene, K.A. Mortier, W.E. Lambert, J. Chromatogr. A 1123 (2006) 71.

[41] EPA, US Code of Federal Regulations, Title 40, Section 136, Appendix B, 2006, p. 319 